\title{
水热制备锌、硅、镁、铁等元素掺杂羟基磷灰石及其表征
}

$$
\text { 宋可可 }{ }^{1} \text {, 黄 浩 }{ }^{2} \text {, 鲁梦婕 }{ }^{3} \text {, 杨安春 }{ }^{2} \text {, 翁 } \text { 杰 }^{1,2} \text {, 段 可 }{ }^{4}
$$

(1. 西南交通大学 医学院, 成都 $610031 ; 2$. 西南交通大学 材料科学与工程学院, 材料先进技术重点实验室, 成都 610031 ; 3. 西南医科大学附属医院病理科, 沪州 646000; 4. 西南医科大学附属医院骨与关节外科, 四川省骨科 置入器械研发及应用技术工程实验室, 沪州 646000)

摘 要: 微量元素掺杂是赋予羟基磷灰石材料更多生物学功能的一条有效途径, 但不同元素的掺杂行为尚待进一步 揭示。本研究使用并行水热合成方式，制备分别含有锌、硅、镁、铁、锰、铜、锶、硒、钴的 9 种羟基磷灰石粒子, 并对其物理化学特性进行研究。结果表明, 元素掺杂显著改变了羟基磷灰石粒子的形貌和晶体生长方向, 但不改变 其物相组成和官能团。晶体的(211)和(112)晶面衍射峰的强度均降低, 结晶度下降。元素实际掺杂效率分析结果显 示锰 $>$ 锌 $>$ 镁 $>$ 铁 (三价 $)>$ 锶 $>$ 钴 $>$ 铜 $>$ 硒 $>$ 硅, 与元素的离子半径大小相关。锰、锌、镁三种元素掺杂量较高, 因其离子 半径与其替代的钲离子接近。铜元素掺杂效率较低是由于其在合成溶液中与氨产生络合作用, 硅元素和硒元素则因 几何结构和电荷 $\left(\mathrm{SiO}_{3}{ }^{2-} 、 \mathrm{SeO}_{3}{ }^{2-} / \mathrm{PO}_{4}{ }^{3-}\right)$ 差异导致掺杂率低。本研究揭示了掺杂行为与离子特性之间的联系, 为功能 化着基磷灰石的设计和开发提供有益的参考基础。

关 键 词: 羟基磷灰石; 元素掺杂; 水热合成; 掺杂行为

中图分类号: R318 文献标志码: A

\section{Hydrothermal Preparation and Characterization of Zn, Si, Mg, Fe Doped Hydroxyapatite}

\author{
SONG Keke ${ }^{1}$, HUANG $\mathrm{Hao}^{2}$, LU Mengjie ${ }^{3}$, YANG Anchun ${ }^{2}$, WENG Jie ${ }^{1,2}$, DUAN Ke $^{4}$
}

(1. College of Medicine, Southwest Jiaotong University, Chengdu 610031, China; 2. Key Lab on Advanced Technologies of Materials (MOE), School of Materials Science and Engineering, Southwest Jiaotong University, Chengdu 610031, China; 3. Pathology department, Affiliated Hospital of Southwest Medical University, Luzhou 646000, China; 4. Sichuan Provincial Lab of Orthopaedic Engineering, Department of Orthopaedics, Affiliated Hospital of Southwest Medical University, Luzhou 646000, China)

\begin{abstract}
Doping of trace elements is an effective way to endow hydroxyapatite with more biological functions. But the behaviors of different element doping remained to be further revealed. Here, nine kinds of hydroxyapatite particles containing zinc, silicon, magnesium, iron, manganese, copper, strontium, selenium, and cobalt, respectively, were prepared by parallel hydrothermal synthesis, and their physicochemical properties were studied. The results show that the morphology and crystal growth direction of hydroxyapatite particles are significantly changed, while the phase
\end{abstract}

收稿日期: 2020-11-20；收到修改稿日期：2020-12-28; 网络出版日期：2021-01-25

基金项目: 国家重点研发计划(2016YFB0700803); 广东省重点领域研发计划项目(2019B010941002); 国家自然科学基金 (51572228); 西南医科大学附属医院创业计划(19038)

National Key Research and Development Program (2016YFB0700803); Research and Development Plan Projects in Key Fields of Guangdong Province (2019B010941002); National Natural Science Foundation of China (51572228); Entrepreneurship Plan of Affiliated Hospital of Southwest Medical University (19038)

作者简介: 宋可可(1996-), 女, 硕士研究生. E-mail: 793952151@qq.com

SONG Keke(1996-), female, Master candidate. E-mail: 793952151@qq.com

通信作者：翁 杰，教授.E-mail: jweng@swjtu.cn; 段 可，副教授.E-mail: keduan9@126.com

WENG Jie, professor. E-mail: jweng@swjtu.cn; DUAN Ke, associate professor. E-mail: keduan9@126.com 
composition is maintained. The peak intensity of (211) and (112) plane diffraction peaks decreased, accompanied by decreased crystallinity. Analysis results show that the actual doping efficiency obeys the tendency of manganese $>$ zinc $>$ magnesium $>$ iron (trivalent) $>$ strontium $>$ cobalt $>$ copper $>$ selenium $>$ silicon. Among these elements, doping amounts of $\mathrm{Mn}, \mathrm{Zn}$ and $\mathrm{Mg}$ are higher, because their ion radii are closed to that of $\mathrm{Ca}^{2+}$. The low doping efficiency of copper is caused by its complexation with ammonia in synthetic solution, while silicon and selenium are caused by different geometry and charge of $\mathrm{SiO}_{3}{ }^{2-}, \mathrm{SeO}_{3}{ }^{2-}$ from that of $\mathrm{PO}_{4}{ }^{3-}$. This study reveals the reasonable relationship between doping behavior and ion characteristics, providing a useful reference for design and development of functionalized hydroxyapatite.

Key words: hydroxyapatite; element doping; hydrothermal synthesis; doping behavior

着基磷灰石 $\left(\mathrm{Ca}_{10}\left(\mathrm{PO}_{4}\right)_{6}(\mathrm{OH})_{2}\right.$, Hydroxyapatite, $\mathrm{HA}$ ) 是人体骨的主要无机成分, 临床上常用作骨骼 创伤和缺损疾病的治疗 ${ }^{[1]}$ 。除钙和磷之外, 人体骨矿 物中含有的其他微量元素, 对骨骼发育起着重要作 用 ${ }^{[2-4]}$ 。其中, 铜 $(\mathrm{Cu})$ 元素参与胶原的交联, 铜缺乏 将降低骨骼力学性能 ${ }^{[5-6]}$; 锌 $(\mathrm{Zn})$ 是骨骼正常发育所 必需的元素, 骨骼生长迟缓和骨质疏松症与锌缺乏 有关 ${ }^{[7-8]}$; 钴 $(\mathrm{Co})$ 元素则可以以维生素 $\mathrm{B}_{12}$ 和辅酶 $\mathrm{B}_{12}$ 配合物的形式刺激造血功能, 影响骨生长 ${ }^{[9]}$ 。此外, 铜、锌、钴元素均具有诱导血管生成和成骨细胞分化 的能力, 可作为促进骨缺损愈合的补充剂 ${ }^{[10-12]}$ 。硒(Se) 是人体必需的微量元素, 可提高免疫系统的细胞增 殖和抗体的合成水平，从而提高机体免疫功能 ${ }^{[13-14]}$ 。 已有大量文献表明, 铁 $(\mathrm{Fe}) 、$ 镁 $(\mathrm{Mg})$ 、硅 $(\mathrm{Si}) 、$ 锶 $(\mathrm{Sr})$ 等其他微量元素对骨骼的发育和修复也有良好的促 进作用 ${ }^{[15-20]}$ 。因此研究合成元素离子掺杂 HA 可以 模拟骨矿物的化学性质, 赋予合成 HA 更好的生物

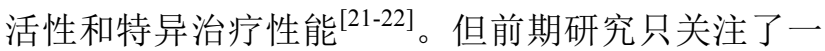
种或两种微量元素掺杂后 HA 的物相及生物学性能 变化, 缺乏对这些元素掺杂特性的系统性研究。因 此, 本研究将对微量元素的掺杂行为, 如进入 HA 晶格的趋势以及对 HA 晶体或粒子的物理化学特性 影响等作深入探究。

水热合成是一种简易的元素掺杂 HA 制备方法, 具有成本低、易操作等优点。然而传统的水热合成 需要高温高压环境, 存在水热烘箱内部温度不均 匀、水热反应釜间温度分布不均匀、反应条件无法 实现真实统一等缺点。因此, 本研究采用六位一体 水热反应装置合成元素掺杂 $\mathrm{HA}$ 粒子。该装置含有 六位可磁力摚拌 $100 \mathrm{~mL}$ 反应器, 通过导热油加热 或模块加热保证加热效率, 针式传感器实时监测反 应温度, 实现温度精控, 从而确保各实验组反应条 件一致。在此基础上可同时并行制备多种单一元素 掺杂的 HA 粒子, 且为后续形貌及物相表征检
测提供一致的实验条件基础。

本研究采用该方法制备了锌、硅、镁、铁、锰、 锶、硒、钴、铜九种元素分别掺杂的 HA 陶瓷粉体 材料, 并对这九种元素掺杂 HA 的行为作了系统探 究, 包括材料表面形貌、物相组成、特征官能团及 掺杂效率等方面的变化。

\section{1 实验方法}

\section{1 材料制备}

在六位一体水热反应装置(西安太康科技有限 公司, PXF-(6))的聚四氟乙烯反应内胆中制备各组 $\mathrm{HA}$ 粉体材料样品。每个容器装入含有一种掺杂元 素(表示为 $\mathrm{M} ; \mathrm{M}=\mathrm{Zn} 、 \mathrm{Mg} 、 \mathrm{Fe} 、 \mathrm{Mn} 、 \mathrm{Sr} 、 \mathrm{Co} 、 \mathrm{Cu}$ 、 $\mathrm{Se} 、 \mathrm{Si})$ 的反应溶液。使用硝酸锌 $\left(\mathrm{Zn}\left(\mathrm{NO}_{3}\right)_{2}\right)$ 、硝酸 镁 $\left(\mathrm{Mg}\left(\mathrm{NO}_{3}\right)_{2}\right)$ 、硝酸铁 $\left(\mathrm{Fe}\left(\mathrm{NO}_{3}\right)_{2}\right)$ 、氯化锰 $\left(\mathrm{MnCl}_{2}\right)$ 、 硝酸铜 $\left(\mathrm{Cu}\left(\mathrm{NO}_{3}\right)_{2}\right)$ 、硝酸锶 $\left(\mathrm{Sr}\left(\mathrm{NO}_{3}\right)_{2}\right)$ 、硝酸钴 $\left(\mathrm{Co}\left(\mathrm{NO}_{3}\right)_{2}\right)$ 作为掺杂元素源, 硅酸钠 $\left(\mathrm{Na}_{2} \mathrm{SiO}_{3}\right)$ 和亚 硒酸钠 $\left(\mathrm{Na}_{2} \mathrm{SeO}_{3}\right)$ 作为硅、硒掺杂剂。将 $0.1 \mathrm{~mol} / \mathrm{L}$ 的硝酸钙溶液 $\left(\mathrm{Ca}\left(\mathrm{NO}_{3}\right)_{2}\right)$ 和掺杂源溶液, 按照 $\mathrm{M} /(\mathrm{Ca}+\mathrm{M})$ (对于 $\mathrm{Zn} 、 \mathrm{Mg} 、 \mathrm{Fe} 、 \mathrm{Mn} 、 \mathrm{Sr} 、 \mathrm{Co} 、 \mathrm{Cu}$ ) 和 $\mathrm{M} /(\mathrm{P}+\mathrm{M})$ (对于 $\mathrm{Si} 、 \mathrm{Se}$ )摩尔比为 $1 \%$ 、3\%或 $5 \%$ 的 比例混合, 之后添加到 $0.1 \mathrm{~mol} / \mathrm{L}$ 的磷酸氢二钠 $\left(\mathrm{Na}_{2} \mathrm{HPO}_{4}\right)$ 溶液中, 保持 $(\mathrm{Ca}+\mathrm{M}) / \mathrm{P}=1.67$ 或 $\mathrm{Ca} /(\mathrm{P}+\mathrm{M})=$ 1.67 。在磁力摚拌下向混合溶液添加硝酸 $\left(\mathrm{HNO}_{3}\right)$ $(10.8 \mathrm{~mol} / \mathrm{L})$ 和尿素 $\left(\mathrm{CON}_{2} \mathrm{H}_{4}\right)(0.56 \mathrm{~mol} / \mathrm{L})$, 将溶液 的 $\mathrm{pH}$ 调节到 2 3。形成透明的混合溶液后, 调节水 热反应装置加热到 $150{ }^{\circ} \mathrm{C}$ 并保温 $3 \mathrm{~h}$ 。高温高压环境 导致尿素分解、pH 增加至 10 11, 使 HA 发生结晶。 反应结束后冷却至室温, 离心(4000 r/min, $2 \mathrm{~min}$ )收 集产品, 去离子水反复冲洗并干燥 $\left(70{ }^{\circ} \mathrm{C}, 48 \mathrm{~h}\right)$ 。根 据加入到反应溶液中的元素掺杂浓度, 产物分别命 名为 $1 \%$ M-HA、 $3 \%$ M-HA 和 5\% M-HA。使用同样 方法合成未掺杂的纯 HA 作为空白对照。所有试剂 
从长征试剂公司(中国成都)购买, 研究过程中均使 用去离子水。

\section{2 材料表征}

使用扫描电子显微镜(SEM, FEI-Quanta-200)对 样品表面形貌进行分析。采用 $\mathrm{X}$ 射线衍射 $(X R D$, Panalyticx'Pert, $\mathrm{Cu}-\mathrm{K} \alpha, 35 \mathrm{~mA}, 45 \mathrm{kV}$ ) 研究样品相组 成并分析样品的结晶度。采用傅里叶红外光谱仪 (FT-IR, Thermo Nicolet5700)检测样品中所含官能 团。采用电感耦合等离子体原子发射光谱(ICP-AES, Spectro Arcos, Speicher)对掺杂元素进行定量检测。 根据公式(1)计算每种元素的相对掺杂率 $(R)$ 。

$$
R=\frac{\chi_{\mathrm{a}}}{\chi_{\mathrm{t}}}
$$

其中, $\chi_{\mathrm{a}}$ 为样品中元素的实际掺杂量; $\chi_{\mathrm{t}}$ 为样品中元 素理论掺杂量。

\section{2 结果与讨论}

\section{1 掺杂元素对产物形貌和晶相结构的影响}

通过 SEM 对合成样品的表面形貌进行观察。未 掺杂的纯 HA 表现出明显的条带状形态(图 1(j1)), 与先前的报告一致 ${ }^{[6]}$ 。掺杂的 HA 样品均显示出不 同程度的形貌变化, 且与元素掺杂浓度相关。其中, $\mathrm{Zn}-\mathrm{HA}$ 和 $\mathrm{Mg}$-HA 由条带状和球状晶体组成, 随着 元素掺杂浓度增加, Zn-HA 的球形直径从 2 5 $\mu \mathrm{m}$ 增 大到 $8 \sim 10 \mu \mathrm{m}$, 形态趋向单一, 球形尺寸逐渐均匀

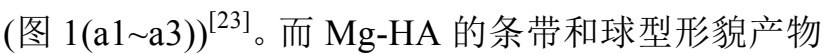
含量比例发生变化, 球状颗粒数量趋向增加但直径 减小(图 1(c1 c3))。结合 $\mathrm{Zn}$ 和 $\mathrm{Mg}$ 掺杂 $\mathrm{HA}$ 形貌发 现, 掺杂元素量增加过程中, 元素趋向占据全部晶 胞的一个 $\mathrm{Ca}$ 位点后再取代第二个。因此会优先改 变粒子形态, 在全部形貌都转变后, 再发生晶体长 大的现象 ${ }^{[24]}$ 。 $1 \% \mathrm{Fe}-\mathrm{HA}($ 图 1(d1 d 3$)$ ) 和 1\%Si-HA (图 1(b1 b3)) 由带状晶体和球形颗粒组成, 但随着 反应溶液中掺杂离子浓度的增加, 球形颗粒减少。 特殊的是, Mn-HA 呈现不规则块状颗粒(图 1(e1 e3)), 颗粒尺寸随着 $\mathrm{Mn}^{2+}$ 浓度的增加而减小至 10 12 $\mu \mathrm{m}$ 。 对于 Sr-HA、Se-HA、Co-HA 三种元素掺杂 HA 样 品, 可观察到随着掺杂离子浓度增加, 形貌逐渐从 与纯 HA 相同的条带状转变为放射花瓣状, 且有继 续堆叠形成球状颗粒的趋势(图 1(f1 f3)，1(g1 g3), 1(h1 h3))。Cu-HA 同样由条带状和球形颗粒组成, 球形颗粒随着 $\mathrm{Cu}^{2+}$ 含量的增加而变大(图 1(i1 i3)), 但形状不规则。

通过 XRD 分析元素掺杂 HA 样品的晶体结构、
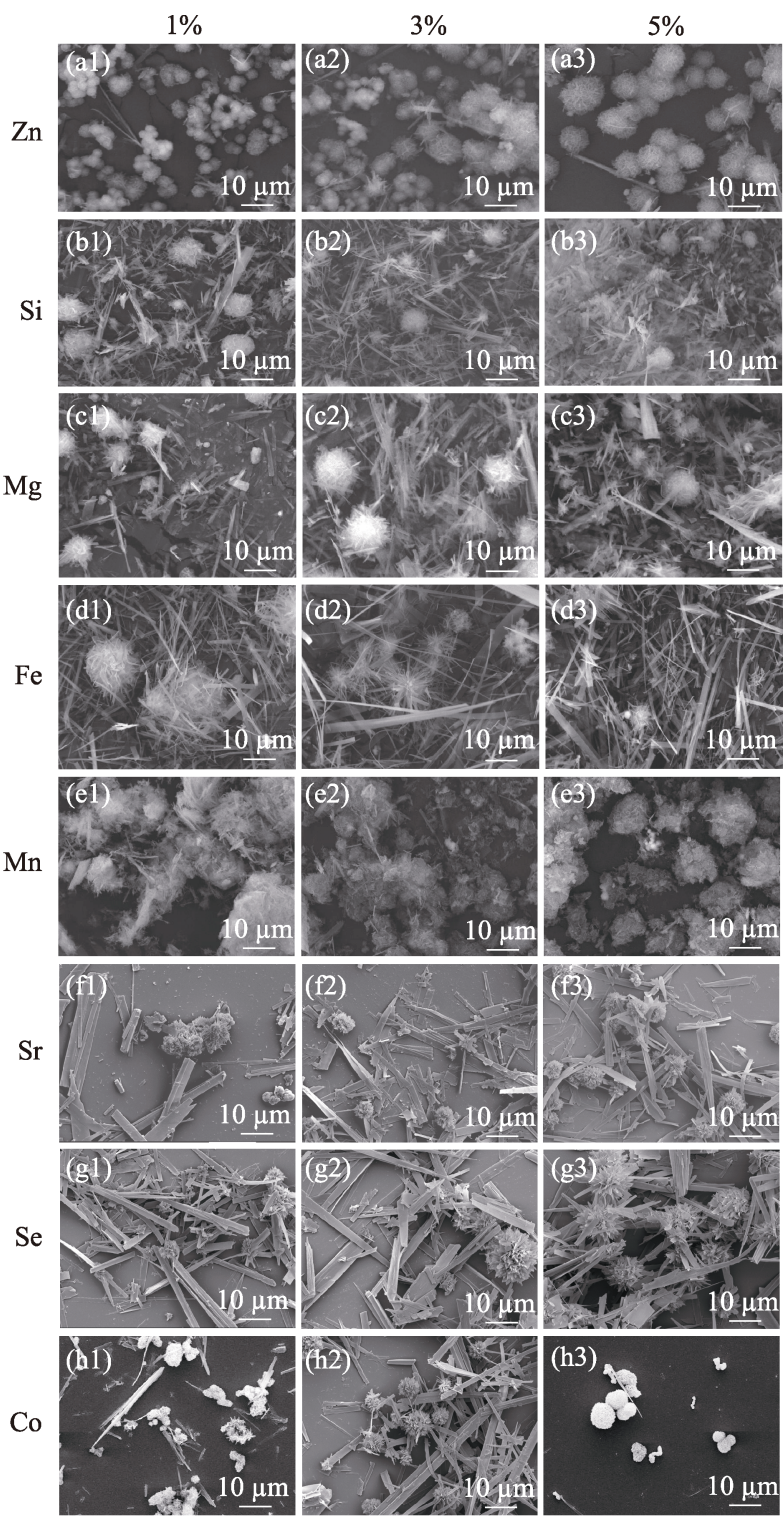

$\mathrm{Cu}$
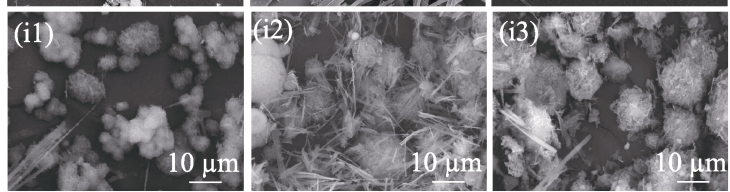

HA

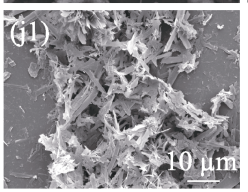

图 $11 \% 、 3 \%$ 和 $5 \%$ 不同元素掺杂 $\mathrm{HA}$ 样品的 SEM 照片

Fig. 1 SEM images of hydroxyapatite samples doped by different elements at $1 \%, 3 \%$ and $5 \%$ doping amount

(a1-a3) Zn-HA; (b1-b3) Si-HA; (c1-c3) Mg-HA; (d1-d3) Fe-HA; (e1e3) Mn-HA; (f1-f3) Sr-HA; (g1-g3) Se-HA; (h1-h3) Co-HA; (i1-i3) $\mathrm{Cu}-\mathrm{HA} ;(\mathrm{j} 1) \mathrm{HA}$

生长方向及结晶度变化。5\%元素掺杂 $[\mathrm{M} /(\mathrm{M}+\mathrm{Ca})$ 或 $\mathrm{M} /(\mathrm{P}+\mathrm{M})=5 \%] \mathrm{HA}$ 合成产物的 XRD 图谱(图 2)显示, 所有样品主要物相结构均为 HA, 与 HA 标准卡片 (JCPDS 09-0432) ${ }^{[24]}$ 特征峰的位置一致。5\%元素掺 杂的各样品衍射峰半峰宽有不同程度的增大，结晶度 有所降低，与 MDI Jade 6 软件分析结果一致(表 1)。 

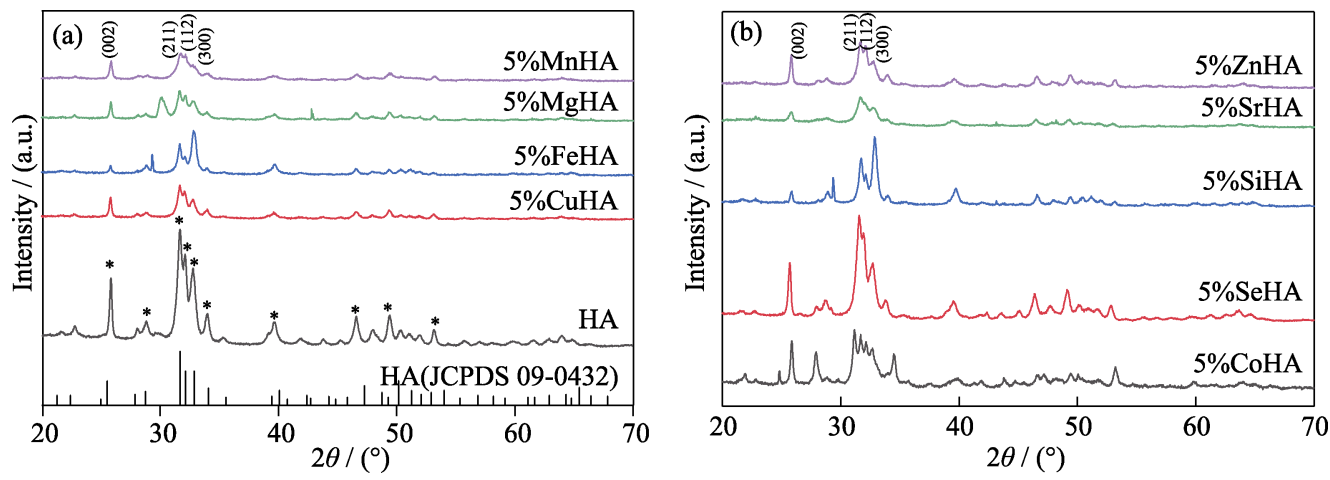

图 $25 \%$ 元素掺杂的样品在 $2 \theta=20^{\circ} \sim 70^{\circ}$ 范围内的 XRD 图谱

Fig. 2 XRD patterns from $2 \theta=20^{\circ}$ to $70^{\circ}$ of hydroxyapatite samples with $5 \%$ doping amount

表 $15 \%$ 元素掺杂样品的结晶度及晶格参数

Table 1 Crystallinity and lattice parameters of $5 \%$ element doped samples $(1 \AA=0.1 \mathrm{~nm})$

\begin{tabular}{ccccc}
\hline Sample & Crystallinity $/ \%$ & $a / \AA$ & $b / \AA$ & $c / \AA$ \\
\hline HA & 97.24 & 9.44 & 9.44 & 6.89 \\
5\%Zn-HA & 82.60 & 9.45 & 9.45 & 6.88 \\
5\%Si-HA & 95.19 & 9.43 & 9.43 & 6.88 \\
5\%Mg-HA & 85.75 & 9.45 & 9.45 & 6.88 \\
5\%Fe-HA & 95.40 & 9.44 & 9.44 & 6.89 \\
5\%Mn-HA & 94.01 & 9.42 & 9.42 & 6.87 \\
5\%Sr-HA & 85.37 & 9.44 & 9.44 & 6.90 \\
5\%Se-HA & 92.85 & 9.43 & 9.43 & 6.88 \\
5\%Co-HA & 92.31 & 9.43 & 9.43 & 6.88 \\
5\%Cu-HA & 91.15 & 9.45 & 9.45 & 6.88 \\
\hline
\end{tabular}

通过对比发现, $5 \% \mathrm{Fe}-\mathrm{HA}$ 和 $5 \% \mathrm{Si}-\mathrm{HA}$ 相较 $\mathrm{HA}$ 和其 他元素掺杂的 HA(图 2)有着更高的 $I(300) / I(211)$ 峰 值强度比, 表明它们的晶体沿着 $a$ 轴优先生长, 显 示出条带状形貌 ${ }^{[23]}$ 。5\% Zn-HA、5\% Se-HA 和 $5 \% \mathrm{Cu}-\mathrm{HA}$ 的峰形及峰强类似, $I(002) / I(300)$ 比值高 于 $\mathrm{HA}, c$ 面晶体发育形成片层结构并堆叠形成球状 形貌。结合 XRD 图谱和 SEM 照片研究发现, 水热 制备元素掺杂 HA 会改变其表面形态和晶体生长方 向，同时有少量其他中间产物出现，但不影响其基
础物相。

\section{2 掺杂离子半径与 HA 官能团和掺杂率的} 关系

采用 FT-IR 分析元素掺杂 HA 的官能团。由图 3 可见, 所有样品所含特征峰无明显差别, 均出现 HA 的官能团特征峰, 说明元素掺杂不对 HA 官能团 构成影响。3400 3700 $\mathrm{cm}^{-1}$ 范围内的宽峰为材料表 面吸附的 $\mathrm{H}_{2} \mathrm{O}$ 分子中 $\mathrm{H}-\mathrm{O}$ 拉伸振动峰, 弯曲振动峰 位于 $1639 \mathrm{~cm}^{-1}$ 处。元素掺杂 $\mathrm{HA}$ 全部显示 $\mathrm{PO}_{4}{ }^{3-}$ 中 $\mathrm{P}-\mathrm{O}$ 的弯曲和拉伸振动峰, 分别位于 564、602、 $958 \mathrm{~cm}^{-1}$ 和 $1034 \mathrm{~cm}^{-1}$ 位置 ${ }^{[25]}$ 。此外, 样品在 872 、 1416 和 $1453 \mathrm{~cm}^{-1}$ 位置出现少量 $\mathrm{CO}_{3}{ }^{2-}$ 的 $\mathrm{C}-\mathrm{O}$ 特征 峰, 形成了部分碳酸根取代的碳酸磷灰石, 且结合 文献[26]得知, 碳酸磷灰石生物相容性良好, 不影 响掺杂 HA 的生物学功效。

掺杂率与掺杂离子的半径和其他特性相关。如 表 2 所示, 除 $\mathrm{Cu}$ 和 $\mathrm{Co}$ 以外的阳离子掺杂元素, 其 离子半径越接近 $\mathrm{Ca}^{2+}$ 的半径, 相对掺杂率越高。表 明尺寸匹配是决定掺杂离子进入 $\mathrm{HA}$ 晶格能力的主 要因素, 与替代掺杂理论的解释相同 ${ }^{[27]}$ 。而 $\mathrm{Co}^{2+}$ 由 于氧化反应而影响掺杂行为, 是除离子半径之外的 特殊因素。 $\mathrm{Fe}^{3+}$ 没有因价态差异而无法掺杂进入
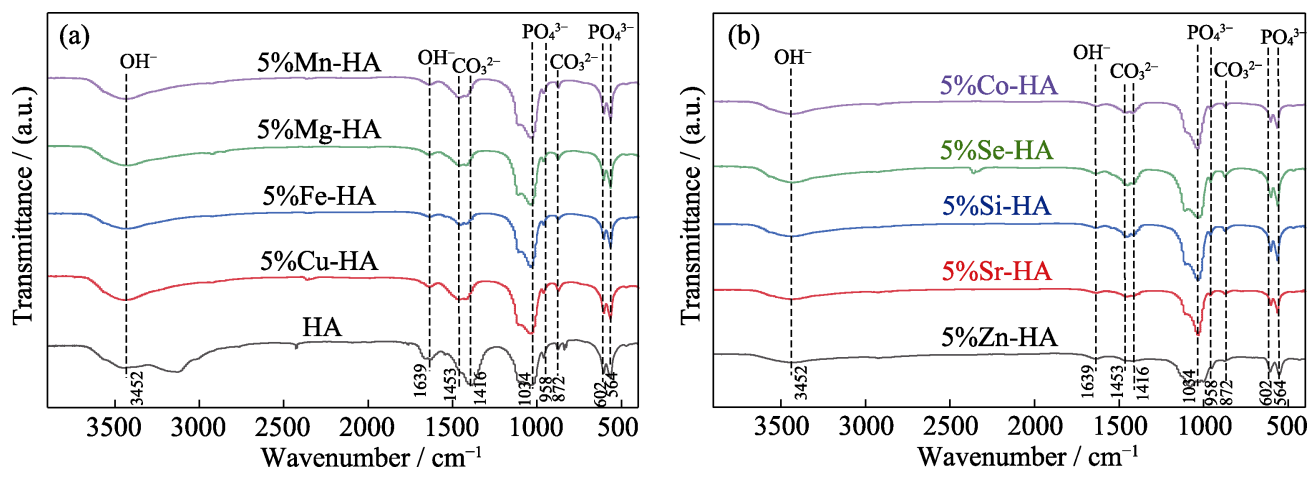

图 $35 \%$ 不同元素掺杂 HA 样品官能团的 FT-IR 光谱图

Fig. 3 FT-IR spectra of functional groups of hydroxyapatites doped by different elements at $5 \%$ doping amount 


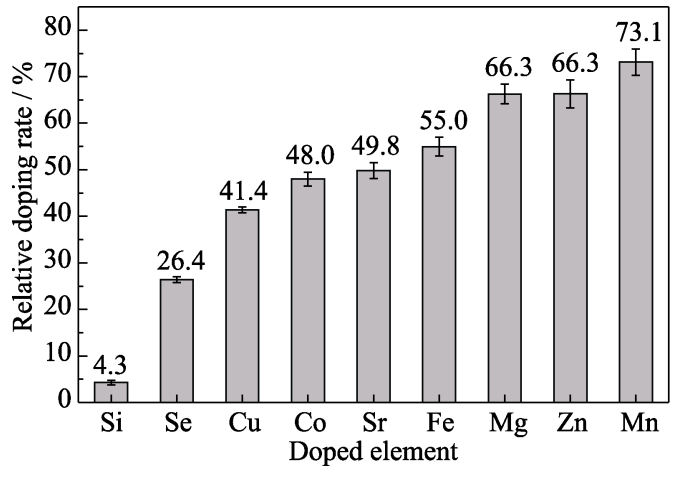

图 $45 \%$ 元素掺杂样品的实际掺杂效率

Fig. 4 Actual doping efficiency of hydroxyapatite doped by different element at $5 \%$ doping amount

表 $2 \mathrm{Ca}^{2+}$ 和所掺杂阳离子的离子(原子)半径尺寸

Table 2 Ion (atom) radius size of doped elements

\begin{tabular}{cc}
\hline Ion & Radius dimension $/ \mathrm{nm}$ \\
\hline $\mathrm{Ca}^{2+}$ & 0.099 \\
$\mathrm{Sr}^{2+}$ & 0.113 \\
$\mathrm{Mn}^{2+}$ & 0.080 \\
$\mathrm{Co}^{2+}$ & 0.074 \\
$\mathrm{Zn}^{2+}$ & 0.074 \\
$\mathrm{Cu}^{2+}$ & 0.072 \\
$\mathrm{Mg}^{2+}$ & 0.065 \\
$\mathrm{Fe}^{3+}$ & 0.064 \\
\hline
\end{tabular}

$\mathrm{HA}$ 中, 也没有实质性地改变其晶体形态, 其根本 原因尚不清楚, 仍有待进一步研究。尽管 $\mathrm{Zn}^{2+}$ 与 $\mathrm{Ca}^{2+}$ 的半径更接近, 但 $\mathrm{Zn}-\mathrm{HA}$ 和 $\mathrm{Mg}-\mathrm{HA}$ 的相对掺 杂率几乎相同, 反应溶液中的尿素在反应过程中分 解生成的 $\mathrm{NH}_{3}$, 会络合游离的 $\mathrm{Zn}^{2+}$, 从而降低掺杂 进入 $\mathrm{HA}$ 的 $\mathrm{Zn}^{2+}$ 浓度。同样, $\mathrm{NH}_{3}$ 与 $\mathrm{Cu}^{2+}$ 之间的强络 合作用也解释了 $\mathrm{Cu}-\mathrm{HA}$ 的相对掺杂率明显较低 ${ }^{[28]}$ 的 原因。硅元素和硒元素分别以硅酸盐阴离子 $\left(\mathrm{SiO}_{3}{ }^{2-}\right)$ 和亚硒酸盐阴离子 $\left(\mathrm{SeO}_{3}{ }^{2-}\right)$ 的形式存在 ${ }^{[29]}$, 在 $\mathrm{HA}$ 中 的掺杂率较低。首先, 可能由于 $\mathrm{Si}$ 与 $\mathrm{C}$ 为同主族元 素, $\mathrm{SiO}_{3}{ }^{2-}$ 代替 $\mathrm{PO}_{4}{ }^{2-}$ 的情况与形成碳酸磷灰石的情 况近似, $\mathrm{SiO}_{3}{ }^{2-}$ 与 $\mathrm{CO}_{3}{ }^{2-}$ 同带负二价电荷且为三角雉 体构型 ${ }^{[30]}$ 。因此推测, $\mathrm{SeO}_{3}{ }^{2-}$ 和 $\mathrm{SiO}_{3}{ }^{2-}$ 偏离了在 $\mathrm{HA}$ 晶体中所替代的 $\mathrm{PO}_{4}{ }^{3-}$ 的负三价电荷及四面体几何 结构(图 5), 从而在与相邻的 $\mathrm{Ca}^{2+}$ 配位时会产生不可 避免的位错。其次, 由于 $\mathrm{pH}$ 的变化, $\mathrm{SiO}_{3}{ }^{2-}$ 会自动 缩合成低聚阴离子, 这些阴离子与 $\mathrm{HA}$ 晶格中的几 何构型不匹配, 导致其结合较差, 因此 $\mathrm{Si}$ 的元素掺 杂效率较 Se 更低。在 HA 材料中掺杂的功能化氧阴 离子相对较少, 因此本研究仅对 $\mathrm{Si}$ 和 $\mathrm{Se}$ 作了研究。 然而, 对阴离子掺杂 $\mathrm{HA}$ (包括非氧阴离子如 $\mathrm{Cl}^{-}, \mathrm{F}^{-}$ 等)需要在后续研究中开展。
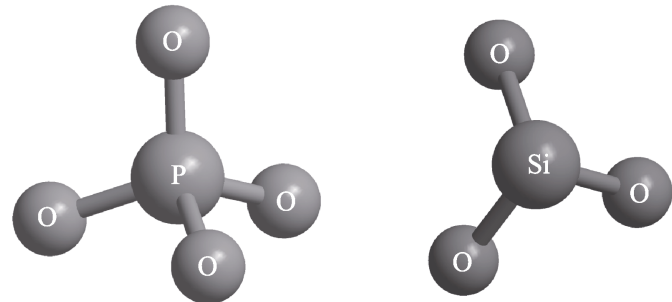

图 5 磷酸根离子和正硅酸根离子的空间构型

Fig. 5 Spatial configurations of phosphate ion and $n$-silicate ion

\section{3 结论}

通过六位一体平行水热合成装置，合成并研究 了锌、硅、镁、铁、锰、铜、硒、锶、钴九种元素 在 HA 中的掺杂行为及其对产物特性的影响。九种元 素均成功掺杂, 进入 HA 晶格。元素掺杂影响了晶体 粒子的形貌, 降低了结晶度, 但不改变其 HA 物相结 构和特征官能团。元素相对掺杂率的大小排序为: $\mathrm{Mn}>\mathrm{Zn}>\mathrm{Mg}>\mathrm{Fe}$ (三价) $>\mathrm{Sr}>\mathrm{Co}>\mathrm{Cu}>\mathrm{Se}>\mathrm{Si}$ ，其主要的 决定因素为掺杂离子半径与 $\mathrm{Ca}^{2+}$ 半径的尺寸差异。 此外, $\mathrm{Co}$ 的氧化反应、 $\mathrm{Cu}$ 与氨的络合作用也分别影 响其掺杂行为, $\mathrm{Si}$ 和 $\mathrm{Se}$ 的实际掺杂量较低是由于 $\mathrm{SiO}_{3}{ }^{2-}$ 和 $\mathrm{SeO}_{3}{ }^{2-}$ 与 $\mathrm{HA}$ 中 $\mathrm{PO}_{4}{ }^{3-}$ 基团的电荷及空间构 型差异。本研究主要涉及金属阳离子和部分含氧阴 离子元素, 对其他元素如稀土元素、卤族元素等特殊 元素掺杂还有待探究。本研究针对元素与掺杂行为 关系的研究，揭示了 HA 掺杂与离子特性之间的联 系, 为设计和开发功能性元素掺杂的 $\mathrm{HA}$ 基材料提 供了理论支持。

\section{参考文献:}

[1] BOANINI E, GAZZANO M, BIGI A. Ionic substitutions in calcium phosphates synthesized at low temperature. Acta Biomaterialia, 2010, 6(6): 1882-1894.

[2] LAKHKAR N J, LEE I H, KIM H W, et al. Bone formation controlled by biologically relevant inorganic ions: role and controlled delivery from phosphate-based glasses. Advanced Drug Delivery Reviews, 2013, 65(4): 405-420.

[3] CHENG Y, WANG M, WANG X X, et al. Investigation on in vitro osteogenic properties of malti-doped hydroxyapatite with natural bone content. Journal of Inorganic Materials, 2016, 31(12): 1341-1346.

[4] 王丽萍. 微量氟、锌、锶掺杂羟基磷灰石晶体结构及生物学效 应研究. 上海: 中国科学院大学(中国科学院上海硅酸盐研究所) 博士学位论文, 2018.

[5] GOMES S, VICHERY C, STEPHANE D, et al. Cu-doping of calcium phosphate bioceramics: from mechanism to the control of cytotoxicity. Acta Biomaterialia, 2018, 65(1): 462-474.

[6] XIAO D Q, TAN Z, FU Y, et al. Hydrothermal synthesis of hollow hydroxyapatite microspheres with nano-structured surface assisted by inositol hexakisphosphate. Ceramics International, 2014, 40(7): 
10183-10188.

[7] QIAO Y, ZHANG W, TIAN P, et al. Stimulation of bone growth following zinc incorporation into biomaterials. Biomaterials, 2014, 35(25): 6882-6897.

[8] KIM H, MONDAL S, BHARATHIRAJA S, et al. Optimized Zn-doped hydroxyapatite/doxorubicin bioceramics system for efficient drug delivery and tissue engineering application. Ceramics International, 2017, 44(6): 6062-6071.

[9] 颜世铭. 钴的生理作用及其与健康的关系. 广东微量元素科学, 2008, 15(7): 19.

[10] BEJARANO J, DETSCH R, BOCCACCINI A R, et al. PDLLA scaffolds with $\mathrm{Cu}$ - and $\mathrm{Zn}$-doped bioactive glasses having multifunctional properties for bone regeneration. Journal of Biomedical Materials Research Part A, 2017, 105A(3): 746-756.

[11] IGNJATOVIC N L, AJDUKOVI Z, RAJKOVI J, et al. Enhanced osteogenesis of nanosized cobalt-substituted hydroxyapatite. Journal of Bionic Engineering, 2015, 12(4): 604-612.

[12] ZHENG Y, YANG Y, DENG Y. Dual therapeutic cobalt-incorporated bioceramics accelerate bone tissue regeneration. Materials \& Engineering C, Materials for Biological Applications, 2019, 99: 770-782.

[13] LI X, WANG Y, CHEN Y, et al. Hierarchically constructed selenium-doped bone-mimetic nanoparticles promote ROS-mediated autophagy and apoptosis for bone tumor inhibition. Biomaterials, 2020, 257: 120253

[14] WANG Y, WANG J, HAO H, et al. In vitro and in vivo mechanism of bone tumor inhibition by selenium-doped bone mineral nanoparticles. ACS Nano, 2016, 10(11): 9927.

[15] PORTER A E, BEST S M, BONFIELD W. Ultrastructural comparison of hydroxyapatite and silicon-substituted hydroxyapatite for biomedical applications. Journal of Biomedical Materials Research Part A, 2004, 68A(1): 133-141.

[16] BENGIY, AMMAR Z A, ZAFER E. Co-doped hydroxyapatites as potential materials for biomedical applications. Microchemical Journal, 2019, 144: 443-453.

[17] 代钊, 汪大林. 锶取代羟基磷灰石的制备方法和生物学特征. 中国组织工程研究, 2018, 22(6): 938-944.

[18] VARITSARA B, XIAOAN Z, LI T, et al. Silicate-based bioceramic scaffolds for dual-lineage regeneration of osteochondral defect. Biomaterials, 2019, 192: 323-333.

[19] DJORDJE V, TAMARA M, TANJA S, et al. $\mathrm{Mg} / \mathrm{Cu}$ co-substituted hydroxyapatite-biocompatibility, mechanical properties and antimi- crobial activity. Ceramics International, 2019, 45(17): 22029-22039.

[20] LIN K, XIA L, LI H, et al. Enhanced osteoporotic bone regeneration by strontium-substituted calcium silicate bioactive ceramics. Biomaterials, 2013, 34(38): 10028-10042.

[21] DING Q Q, ZHANG X J, HUANG Y, et al. In vitro cytocompatibility and corrosion resistance of zinc-doped hydroxyapatite coatings on a titanium substrate. Journal of Materials Science, 2015, 50(1): 189-202.

[22] ALSHEMARY A Z, GOH YF, AKRAM MU, et al. Barium and fluorine doped synthetic hydroxyapatite: characterization and in-vitro bioactivity analysis. Science of Advanced Materials, 2015, 50: $189-202$.

[23] NOURI-FELEKORI M, KHAKBIZ M, NEZAFATI N. Synthesis and characterization of $\mathrm{Mg}, \mathrm{Zn}$ and Sr-incorporated hydroxyapatite whiskers by hydrothermal method. Materials Letters, 2019, 243(MAY15): 120-124.

[24] WANG Q, LI P, TANG P, et al. Experimental and simulation studies of strontium/fluoride-codoped hydroxyapatite nanoparticles with osteogenic and antibacterial activities. Colloids and Surfaces B: Biointerfaces, 2019, 182: 110359.

[25] KIM H, MONDAL S, BHARATHIRAJA S, et al. Optimized Zn-doped hydroxyapatite/doxorubicin bioceramics system for efficient drug delivery and tissue engineering application. Ceramics International, 2018, 44(6): 6062-6071.

[26] MA X, PENG W, SU W, et al. Delicate assembly of ultrathin hydroxyapatite nanobelts with nanoneedles directed by dissolved cellulose. Inorganic Chemistry, 2018, 57: 4516-4523.

[27] KARUNAKARAN G, KUMAR G S, CHO E B, et al. Microwaveassisted hydrothermal synthesis of mesoporous carbonated hydroxyapatite with tunable nanoscale characteristics for biomedical applications. Ceramics International, 2019, 45(1): 970-977.

[28] MONIKA S. Substituted hydroxyapatites for biomedical applications: a review. Ceramics International, 2015, 41(8): 9203-9231.

[29] TOUNSI H, DJEMAl S, PETITTO C, et al. Copper loaded hydroxyapatite catalyst for selective catalytic reduction of nitric oxide with ammonia. Applied Catalysis B Environmental, 2011, 107(1): 158-163.

[30] HE LEI, LI HONGYU, CHEN XINGYU, et al. Selenium-substituted hydroxyapatite particles with regulated microstructures for osteogenic differentiation and anti-tumor effects. Ceramics International, 2019, 45(11): 13787-13789. 